\title{
Síndrome da resposta inflamatória sistêmica na circulação extracorpórea: papel das interleucinas*
}

\author{
Helmiton Vieira de MOURA**, Pablo M. A. POMERANTZEFF, Walter José GOMES
}

RBCCV 44205-564

Moura H V, Pomerantzeff P M A, Gomes W J - Síndrome da resposta inflamatória sistêmica na circulação extracorpórea: papel das interleucinas. Rev Bras Cir Cardiovasc 2001; 16(4): 376-87.

RESUMO: A síndrome da resposta inflamatória sistêmica (SIRS) na cirurgia cardíaca ocorre, com maior freqüência, com o uso da CEC. Isto se deve ao contato do sangue com superfícies não endoteliais e, mais tarde, à reperfusão. Esse contato leva à liberação de componentes moleculares, dos quais as anafilotoxinas ativadas pelo fator de complemento, que estimulam a liberação de citocinas pró-inflamatórias, tais como a IL-1, IL-6, FNT-a. Estas interleucinas são responsáveis por indução de febre, neutrofilia, com aumento da adesividade entre neutrófilos e miócitos e/ou endotélio, e agem de forma sinergética estimulando a produção de outras interleucinas pelos monócitos e leucócitos (IL-6, IL-8). Observa-se que a isquemia intestinal, decorrente do uso da CEC, ocasiona a produção de IL-6, FNT-a, IL-1 no epitélio intestinal, e a reperfusão pós-CEC provoca a expressão de IL-1 e FNT-a no endotélio microvascular e pode gerar uma disfunção que danifica os cardiomiócitos. Seguindo a cascata de reações pós- CEC, a síndrome do distress respiratório do adulto é mediada pela IL-8, encontrada a nível alveolar. Cérebro e fígado também podem ser afetados pelos fenômenos causados pela isquemia/reperfusão e se demonstra que as interlucinas, uma vez mais (IL-8 e IL-13, respectivamente), têm um papel importante na série de eventos que ocorre. As técnicas de CEC bem como a anestesia podem influenciar a atividade das interleucinas. Não há um consenso sobre terapias preventivas ou de tratamento das complicações advindas da SIRS. Intervenção a nível dos mediadores da inflamação, procedimento de descontaminação digestiva, uso de drogas como a indometacina ou esteróides são opções possíveis. A regulação do processo inflamatório, de um modo geral, depende do equilíbrio entre citocinas pró- e anti-inflamatórias. Estas últimas são detectáveis no indivíduo normal, enquanto que as pró-inflamatórias apenas se manifestam localmente ou são quase inexistentes. Conclui-se que, o aumento da concentração das interleucinas pró-inflamatórias no organismo é um fato esperado, em grandes cirurgias, e que trará complicações se este aumento for exacerbado e se o organismo estiver muito debilitado.

DESCRITORES: Circulação extracorpórea, efeitos adversos. Interleucinas. Procedimentos cirúrgicos cardíacos, efeitos adversos.

\section{OBJETIVOS}

Em seguida será apresentado o papel das principais interleucinas envolvidas dentro do quadro inflamatório subseqüente à circulação extracorpórea, incluindo, ainda, os danos sistêmicos produzidos por estas interleucinas, bem como suas interações e como se manifestam, com base na visão de vários autores ao longo dos últimos anos.

\footnotetext{
* Monografia de final de curso. Escola Paulista de Medicina da Universidade Federal de São Paulo - UNIFESP. São Paulo, SP, Brasil. Recebido para publicação em setembro de 2001.

**Aluno do curso de Especialização em Perfusão Cardíaca- UNIFESP.

Orientador: Prof. Dr. Pablo Pomerantzeff. Livre-Docente do Departamento de Cardiopneumologia da Universidade de São Paulo - USP. Médico Assistente da Divisão de Cirurgia do Instituto do Coração do Hospital das Clínicas da Faculdade de Medicina da USP.

Professor: Prof. Dr. Walter José Gomes. Livre-Docente da Disciplina de Cirurgia Cardiovascular, Coordenador do curso de Especialização em Perfusão Cardíaca- UNIFESP.

Endereço para correspondência: Helmiton Vieira de Moura. Av. Leôncio de Magalhães, 1314. Jd. São Paulo. São Paulo, SP. CEP 02042-001. Tel: (11) 6959-4426. e-mail: hvmm@hotmail.com
} 
Moura H V, Pomerantzeff P M A, Gomes W J - Síndrome da resposta inflamatória sistêmica na circulação extracorpórea: papel das interleucinas. Rev Bras Cir Cardiovasc 2001; 16(4): 376-87.

INTRODUÇÃO

\section{Breve Histórico da Circulação Extracorpórea}

A evolução da circulação extracorpórea ao longo dos anos foi lenta e gradual, passando de um procedimento extremamente arriscado para um processo sistemático, minucioso, seguro e rotineiro, que vem sendo praticado diariamente em milhares de centros cirúrgicos no mundo todo. Podemos dividir essa evolução em três períodos.

O período conceptual e de desenvolvimento, que compreende os eventos anteriores a 1950, onde John e Mary Gibbon desenvolveram as primeiras máquinas de circulação extracorpórea.

O período de tecnologia aplicada, de 1950 a 1970. Nesse período desenvolveram-se novos oxigenadores e foi, ainda, aplicada a hipotermia moderada por McQuiston, em 1950, para a redução da taxa metabólica. A evolução dos materiais biocompatíveis, a colocação de filtros e cata-bolhas também diminuíram as complicações observadas nos pós-operatórios.

O período de refinamento, que compreende 1970 em diante, onde o uso de novas técnicas cirúrgicas, tecnologia e drogas foram diminuindo os efeitos deletérios desse procedimento ${ }^{(1,2)}$.

Apesar disso, a CEC impõe ao organismo um número de alterações importantes com o desvio do sangue para um circuito artificial, como a mudança do regime do fluxo sangüíneo, possível aumento do gradiente de temperatura, stress mecânico sobre os elementos figurados do sangue devido ao seu contato com superfícies não endoteliais, filtros, compressão, turbulências, etc.

\section{A Resposta Inflamatória Sistêmica}

Todo trauma, grande cirurgia ou queimadura, choque prolongado ou hipotermia induz uma resposta inflamatória com graus variados de severidade, cuja etiologia não está necessariamente ligada à infecção. Esta inflamação é em geral de difícil identificação, pelo fato de não haver um consenso nas definições dos sintomas observados. Com o intuito de compreender melhor o que se entende por inflamação, o American College of Chest Physicians desenvolveu uma série de definições de maneira que o quadro da reação inflamatória poderia ser identificado mais facilmente em seus múltiplos aspectos $(3,4)$.

\section{São elas:}

Infecção: Fenômeno microbiológico caracterizado por uma resposta inflamatória à presença de microrganismos ou a invasão de um tecido hospedeiro normalmente estéril por estes microrganismos. sangue.
SIRS: A resposta inflamatória sistêmica a uma variedade de agressões clínicas severas que se manifesta por duas ou mais destas condições:

1. temperatura corporal $>38^{\circ} \mathrm{C}$ ou $<36^{\circ} \mathrm{C}$;

2. freqüência cardíaca $>90$ batidas por minuto;

3. freqüência respiratória $>20$ inspirações por minuto ou $\mathrm{PaCO}_{2}<32 \mathrm{mmHg}$;

4. contagem de glóbulos brancos $>12000 /$ cumm, < 4000 /cumm ou $>10 \%$ das formas imaturas.

Sepsis: A resposta sistêmica à infecção que se manifesta por duas ou mais das condições supracitadas na definição de SIRS.

Sepsis severa: Sepsis associada com disfunção de orgãos, hipoperfusão ou hipotensão. Hipoperfusão e anormalidades de perfusão podem incluir, mas não estão limitadas a acidose láctica, oligúria ou alteração aguda do estado mental.

Choque séptico: Sepsis induzida com hipotensão e anormalidades de perfusão, apesar de se haver efetuado uma infusão adequada de fluído. As anormalidades incluem mas não estão limitadas a acidose láctica, oligúria ou alteração aguda do estado mental. Pacientes que recebem agentes vasopressores ou inotrópicos podem não ser hipotensivos no momento em que se medem essas anormalidades.

Hipotensão induzida por sepsis: Pressão sistólica sangüínea $<90 \mathrm{mmHg}$ ou uma redução de ${ }^{3} 40 \mathrm{mmHg}$ da linha de base na ausência de outras causas para hipotensão;

Síndrome de disfunção múltipla de órgãos (MODS): Presença de alteração na função de órgãos num paciente com enfermidade aguda de maneira tal que a homeostase não possa ser mantida sem intervenção médica.

Da mesma maneira que os demais procedimentos invasivos, a circulação extracorpórea é traduzida pelo organismo como um agente agressor e desencadeia uma série de reações e mudanças no equilíbrio fisiológico e, entre todas as reações existentes, a resposta inflamatória sistêmica do organismo (SIRS) é a mais complexa e nociva (4-6). O contato do sangue com superfícies não endoteliais é o fator principal que desencadeia esta resposta. Existem ainda outros elementos de extrema importância como - período de isquemia e reperfusão tissular, hipotermia e as manipulações feitas no sistema de coagulação. Ela pode ocorrer sem envolver infecção e manifestar-se desde uma forma muito leve até uma forma exacerbada caracterizada por um quadro clínico que envolve discrasias sangüíneas, retenção de líqüido no espaço intersticial, vasoconstrição, grande sensibilidade à infecções e insuficiência múltipla de órgãos, podendo levar à morte.

Os mecanismos da síndrome começaram a ser estudados no final dos anos 60. Assim, HAIRSTON et 
Moura H V, Pomerantzeff P M A, Gomes W J - Síndrome da resposta inflamatória sistêmica na circulação extracorpórea: papel das interleucinas. Rev Bras Cir Cardiovasc 2001; 16(4): 376-87.

al. (7), em 1969, comprovaram a diminuição das imunoglobulinas e a redução do poder bactericida do soro sangüíneo. PARKER et al. (8), em 1972, descreveram o aumento da permeabilidade capilar e a formação de edema intersticial causando disfunção de vários órgãos após a circulação extracorpórea. Sugeriram que haveria uma ligação entre a desnaturação das imunoglobulinas, a ativação do complemento e a formação de anafilotoxinas. Também sugeriram que a ativação do complemento, a ativação e adesão plaquetárias e a agregação dos leucócitos estariam associadas à lesão da membrana endotelial durante a perfusão, levando ao aumento da permeabilidade renal e possível disfunção renal, hepática e pulmonar (9). Mas foi apenas em 1981 que o caminho para uma melhor compreensão e identificação da resposta inflamatória abriu-se com a descrição da ativação do complemento durante a circulação extracorpórea, por CHENOWETH et al. (10). Observando as similaridades entre os sintomas da reação inflamatória e a atividade biológica das anafilotoxinas $\mathrm{C} 3 \mathrm{a}$ e $\mathrm{C} 5 \mathrm{a}$, eles investigaram a ativação destas porções terminais do complemento pós- operatoriamente através de radioimunoensaios. Nos anos subseqüentes, a cascata de elementos ativados durante as grandes cirurgias e a CEC começaram a ser estudadas isoladamente.

Entretanto, ainda é difícil entender os mecanismos responsáveis pela produção da síndrome completa ou de alguns de seus componentes. Isto se dá porque a resposta do organismo é muito variável e também poucos indivíduos apresentam respostas clinicamente identificadas. Segundo BENNETTGUERRERO (4), é difícil fazer-se uma identificação clínica da síndrome, pelo fato de não haver testes diagnósticos confiáveis que quantifiquem o grau de inflamação sistêmica. E mesmo se já houvesse um, não haveria meios de predizer, em um caso de SIRS mais severa, se haveria insuficiência de algum órgão e qual seria esse órgão. Como terceiro problema, ainda que o diagnóstico seguro fosse possível, não há terapias preventivas ou tratamentos específicos de uso corrente. E estas respostas estão relacionadas à tolerância individual. Por exemplo, crianças de baixo peso, principalmente os recémnatos, os idosos e os pacientes submetidos a longas perfusões são bem mais suscetíveis a desenvolverem essa reação generalizada (2).

Os avanços nas técnicas imunológicas permitem medir tanto o plasma quanto os componentes intracelulares desta resposta perioperatória multifacetada. Apesar de não corresponder, ainda, a um diagnóstico, já se pode avaliar o grau de participação de cada elemento envolvido na resposta inflamatória. Isto inclui ativação do complemento, coagulação, cascatas fibrinolíticas e de kalicreína, ativação de neutrófilos pelas citocinas com degranulação e liberação de protease, produção de radicais de $\mathrm{O}_{2}$ e a síntese de várias citocinas de células mononucleares [TNF, IL-1, IL-6 (11), IL-2, IL4 , IL-5, IL-8 ${ }^{(12)}$, IL-12 $\left.{ }^{(13)}, \mathrm{IL}-13^{(14)}\right]$.
Os Diversos Componentes Moleculares Envolvidos na SIRS

Como já descrito anteriormente, o que desencadeia toda a resposta inflamatória é o contato do sangue com as superfícies do circuito da CEC. Suas superfícies não endoteliais, assim como os microagregados presentes nos hemodiluentes, acionam um mecanismo de defesa contra agentes estranhos ao organismo.

Em continuidade, o endotélio vascular responde à agressão representada pela canulação e mudanças do regime do fluxo do sangue, que perde o caráter pulsátil por um lado, e por outro apresentará turbulência localizada por causa da CEC. Este conjunto de condições ativa uma série de sistemas imunoreguladores e coagulantes que não se limitam a agir localmente. São eles:

1. Coagulação- a heparinização sistêmica inibe a fase final da coagulação sangüínea, mas o mesmo não acontece na sua fase inicial, ativada pelo fator XII. Ainda que não haja formação de coágulos, certos fatores penetram na microcirculação tais como a fibrina, que contribui para a reação inflamatória, ou o fibrinogênio, que é adsorvido nas superfícies internas do circuito de CEC, assim como outros fatores da coagulação, como as gamaglobulinas e lipoproteínas. Além disso, muitas plaquetas aderem à superfície do circuito e aí permanecem inativas. Aquelas que não são seqüestradas podem danificar-se, liberando um potente vasoconstritor na circulação, o tromboxano $\mathrm{A}$.

2. Calicreína- o fator XII da coagulação é ativado assim que a CEC se inicia, e este fator inicia a cascata de calicreína. Esta induz a formação da bradicinina que, por sua vez, aumenta a permeabilidade vascular e dilata as arteríolas. Os subprodutos da ativação das cininas são proteínas que podem lesar as membranas celulares.

3. Complemento- é ativado indiretamente pelo fator XII da coagulação. A plasmina originada na cascata da coagulação começada pelo fator XII, é um dos elementos estimuladores da expressão do complemento. O sistema do complemento consiste de 20 proteínas plasmáticas que fazem parte do sistema imune normal. As mais importantes delas são a C3a, C3b, C5 e C5a. Sua ativação pode, entretanto, contribuir para reações adversas em certas circunstâncias. Elas aumentam a permeabilidade capilar, são agentes quimiotáticos de neutrófilos e contribuem para a ativação continuada de outros fatores de complemento, como por exemplo, C5, que é ativado pelo C3b. Há dois caminhos de ativação conhecidos para o complemento: o clássico, que pode ser iniciado por um lipídio de endotoxina, certos carbohidratos, bactérias gramnegativas, partículas virais, parasitas e material liberado por células danificadas em casos de trauma ou sepsis; e o alternativo, ativado por bactérias, 
Moura H V, Pomerantzeff P M A, Gomes W J - Síndrome da resposta inflamatória sistêmica na circulação extracorpórea: papel das interleucinas. Rev Bras Cir Cardiovasc 2001; 16(4): 376-87.

fungos, vírus e células infectadas por vírus, células de tumor, certos parasitas e superfícies artificiais como as encontradas na CEC $(2,15)$. Estudos recentes demonstraram que a ação do complemento sobre os mastócitos, macrófagos, miócitos e outras células estimulam a liberação de interleucinas. Estas vêm regular a migração de neutrófilos para os capilares sangüíneos ocasionando uma série de eventos intercelulares tais como a adesão, como a liberação de proteínas de fase aguda a nível hepático, desencadeando toda a sintomatologia da SIRS como ela é reconhecida hoje ${ }^{(15)}$

\section{Interleucinas}

\section{Indução das interleucinas}

\section{A) Interleucinas pró-inflamatórias}

Segundo estudos, a anafilotoxina C5a parece ser a mais danosa durante a ativação maciça do complemento (16). Pequenas quantidades de C5a podem induzir a agregação de neutrófilos e sua aderência ao endotélio vascular e às superfícies estranhas. Da mesma maneira, frações de complemento C3b e sua forma inativa, podem induzir degranulação dos neutrófilos e liberação de interleucina-1b (IL-1) pelos monócitos já aderidos à superfície do circuito da CEC ou ao endotélio vascular. Estudos recentes também relacionam o fator de ativação plaquetária (PAF) com a liberação de interleucinas $(17,18)$.

A IL-1 é um componente importante das respostas inflamatória e imune. Além das anafilotoxinas do complemento, ela pode ser induzida também por endotoxinas (LPS) e por outra citocina, ela mesma induzida por LPS: TNF-a. Febre, neutrofilia e liberação da proteína C-reativa são mediadas pela IL1. Células em cultura tratadas com TNF-a ou IL-1 tornam-se super adesivas em neutrófilos. Essa adesividade ocorre pela expressão da molécula de adesão ICAM-1, induzida pela IL-1 e outras interleucinas. Igualmente, a IL-1 induz a liberação de conteúdo específico dos grânulos de neutrófilos humanos e a síntese de outra interleucina, a IL-6, pelos monócitos.

Produção maior de IL-1 foi encontrada, de modo transiente, depois de CEC, com sua concentração máxima tendo sido atingida a $24 \mathrm{~h}$ pós CEC, coincidindo com o pico de temperatura corporal do paciente (19). Neste estudo, a IL-1 foi assessada medindo-se a atividade funcional e concentração do antígeno de IL-1 em lisatos de monócitos de pacientes durante e depois da CEC. Em contraste, outros estudos não a detectaram no plasma $(20,21)$. Parece que, durante a maior parte das inflamações agudas, ela não é sistematicamente medida. Uma possível explicação para isso está nos diferentes métodos de medida usados e também à presença de inibidores plasmáticos específicos que agem como antagonistas da IL-1. Mesmo essa dificuldade de detecção da IL-1 circulante não excluiria sua produção local. Um estudo de ROTH-ISIGKEIT et al. (22) demonstram que essa flutuação deve estar ligada à hemodiluição e que quando os níveis de citocinas não são corrigidos para levar-se em conta este fato, as concentrações medidas são muito menores e variam muito mais que as corrigidas.

Além da pirogenicidade associada à IL-1, também se sabe que ela é mediadora da migração e da aderência dos neutrófilos aos miócitos, contribuindo para o dano cardíaco durante a reperfusão; IL-1 está presente intra-alveolarmente em pacientes que apresentam a síndrome do stress respiratório no adulto e tem participação na vasodilatação apresentada por pacientes pós CEC.

A IL-1 é responsável pela indução de outras interleucinas e age em sinergia com elas e principalmente com TNF-a no feedback que dá continuidade ao processo. Estudos de BEUTLER \& CERAMI (23), CASEY ${ }^{(24)}$ e CAIN et al. (25) observaram esta sinergia entre a IL-1, TNF, LPS (uma endotoxina) e a IL-6.

Outras duas interleucinas pró-inflamatórias importantes são IL-6 e IL-8, ambas secretadas pelos leucócitos.

A IL-6 é também produzida por monócitos, células endoteliais e linfócitos. A IL-6 está fortemente relacionada à produção e liberação das proteínas de fase aguda dos hepatócitos (proteína reativa $\mathrm{C}$, selectinas) e pode induzir febre e causar a liberação de hormônio adrenocorticotrófico. Ela foi detectada no soro humano depois de queimaduras, operação eletiva e em pacientes sépticos. Depois de cirurgias, a concentração de IL-6 no soro eleva-se dentro de 2 a 4 horas após a incisão e a intensidade da resposta correlaciona-se com a duração da cirurgia. A IL-6 pode ser um bom marcador de dano tissular e seus níveis podem ser um prognóstico de choque séptico pois existe uma correlação comprovada entre suas concentrações e o grau de severidade do quadro clínico do paciente. As concentrações de IL6 respondem à CEC de modo similar, seja com o uso de oxigenador de membrana ou de bolhas. Este padrão de resposta é consistente com o papel de um mediador importante na resposta de fase aguda à $C E C$ e parece ser o indicador mais preciso da evolução do estado do paciente $(24,26)$.

Estudos demonstram que a IL-6 aumenta significativamente no plasma durante as primeiras horas a partir do início da CEC $(4,9,20,27,28)$ e que há uma correlação entre seus níveis plasmáticos e mortalidade em pacientes pós-CEC (quanto mais IL-6 medida, maior a probabilidade de mortalidade). Ela também tem sido detectada em níveis maiores que os níveis basais normais em pacientes portando insuficiência cardíaca congestiva e da mesma ma- 
Moura H V, Pomerantzeff P M A, Gomes W J - Síndrome da resposta inflamatória sistêmica na circulação extracorpórea: papel das interleucinas. Rev Bras Cir Cardiovasc 2001; 16(4): 376-87.

neira, seus níveis foram bons preditores independentes de mortalidade ${ }^{(29)}$. Um estudo experimental de CASEY (24) sugere que a IL-6 seria mais um marcador que um mediador, representando o grau de inflamação ativa das células endoteliais. Curiosamente, parece existir uma correlação inversa entre as concentrações de IL-6 e de lipídios no plasma, o que também poderia explicar a variabilidade dos resultados obtidos de estudo para estudo. O mecanismo proposto para explicar o fato é a neutralização da endotoxina pela fixação de lipídios, sobretudo os de alta densidade

A IL-2 e a IL-12, duas interleucinas menos conhecidas, também podem estar presentes pós CEC. A primeira ativa as células NK (natural killer), pré linfócitos $\mathrm{T}$ que destroem as células alvo por lise, sem comprometimento do anticorpo ou do complemento. A IL-2 é liberada logo após a indução da anestesia; apresentando um valor basal diferente de zero, atinge seu pico após administração de protamina. Segundo um estudo de STEINBERG et al. ${ }^{(9)}$, seu aumento de concentração durante a CEC, embora mensurável, não foi estatisticamente significante em relação aos valores de base. Essa dificuldade na sua detecção pode ser contornada nos últimos tempos através da medida do seu receptor antagonista solúvel, a IL-2ra.

A IL-12, também pró-inflamatória, foi menos estudada até agora. Sabe-se que é induzida em linfócitos e ativa macrófagos. Entretanto, não se expressará quando a IL-10, uma interleucina antiinflamatória, for induzida (13).

A IL-5 também é moduladora da leucotaxia mas não há nenhuma referência sobre sua função durante ou após a CEC.

A IL-8 segue a mesma linha de ação da IL-6 durante e após a $\operatorname{CEC~}(27,28)$, mas não se estabeleceu com clareza seu papel como mediador ou preditor de evolução do quadro clínico do paciente pós cirurgia cardíaca. Sabe-se, entretanto, que ela é um agente de quimiotaxia potente, fazendo neutrófilos e leucócitos se deslocarem para o local de infecção ou trauma. Também induz a degranulação dos neutrófilos.

\section{B) Interleucinas anti-inflamatórias}

Segundo estudos, a regulação do processo inflamatório envolve um equilíbrio entre as citocinas pró e anti-inflamatórias. Estas últimas têm, então, o papel de inibidoras das primeiras.

A mais bem estudada e conhecida dentre elas é a IL-10. Ela inibe a síntese do TNF, IL-1, IL-6 e IL-8 em monócitos e macrófagos. Observa-se um aumento de sua concentração e da do receptor antagonista do IL-1 (IL-1ra) concomitante à diminuição das concentrações das citocinas pró-inflamatórias. Estudos in vitro e in vivo $(13,30)$ mostram que a IL-10 é induzida em linfócitos que infiltram as zonas isquêmicas e infartadas. Ela suprime metaloproteínas e estimula inibidores da metaloproteinase em macrófagos, o que favorece a preservação da matriz celular. Sua indução inicial é dependente da reperfusão.

A IL-4, também expressa em linfócitos, suprime a produção de IL-8, retira os receptores solúveis do TNF dos seus sítios da superfície de certas células. Com relação à CEC, ela apresenta sua concentração máxima em seguida à administração de protamina ${ }^{(9)}$. Ela é responsável pela indução da transcrição da selectina $P$, uma proteína de fase inflamatória (31).

A IL-13 é uma interleucina estreitamente relacionada ao fígado e regula o dano inflamatório do tecido hepático, suprimindo a produção de mediadores pró-inflamatórios dos macrófagos, diminuindo, assim, a atração dos neutrófilos para o local (14).

De um modo geral, a concentração livre de citocinas anti-inflamatórias em indivíduos sadios é considerável, enquanto que as citocinas pró-inflamatórias são quase inexistentes, pois sua atividade fisiológica fica confinada ao local de sua liberação e tem uma vida relativamente curta.

\section{O equilíbrio das citocinas}

Para evitar efeitos indesejáveis das citocinas pró-inflamatórias a nível sistêmico, há um sistema de glicosaminoglicanos endoteliais ligados por membrana que mantém estas interleucinas concentradas no local. Não se consegue medi-las facilmente num local, a não ser nos pulmões, onde se pode detectar as interleucinas na lavagem broncoalveolar. Aí, mediram-se níveis de TNF e IL-1, que se correlacionam com o desenvolvimento da síndrome do stress respiratório no adulto (4). Concentrações plasmáticas destas citocinas não foram capazes de apontar ou prever o desenvolvimento da síndrome.

A CEC representa, com outros eventos clínicos, uma perturbação deste equilíbrio entre citocinas pró e anti-inflamatórias. O interessante é notar que o sistema de contenção, que normalmente mantém as citocinas pró-inflamatórias confinadas, não mais funciona ou este sistema torna-se menos eficaz sob as condições impostas pela circulação extracorpórea, razão pela qual a sua ação atinge níveis sistêmicos. O seu papel dentro do quadro da CEC será detaIhado a seguir.

O papel das interleucinas na reação inflamatória sistêmica durante a CEC

Durante a CEC, o organismo reage ao desvio do sangue para o circuito coração-pulmão artificial, do mesmo modo que reagiria perante um episódio de hemorragia. O intestino, detectando uma suposta 
Moura H V, Pomerantzeff P M A, Gomes W J - Síndrome da resposta inflamatória sistêmica na circulação extracorpórea: papel das interleucinas. Rev Bras Cir Cardiovasc 2001; 16(4): 376-87.

hemorragia, entra em vasoconstrição periférica, para que haja proteção dos órgãos nobres, coração e cérebro, ocorrendo, assim, um shunt preferencial. Essa hipoperfusão pode progredir para isquemia, resultando em complicações condizentes com choque circulatório, que pode levar à perda da função de barreira da mucosa intestinal. A perda ou diminuição da função de barreira implica em que o conteúdo da flora intestinal pode translocar-se e entrar na circulação sistêmica. Esta situação origina o chamado choque endotóxico. Há crescente evidência de que este estado é resultado da geração de citocinas pelo intestino sob isquemia. Estudos in vitro demonstram que o epitélio intestinal isquêmico torna-se um produtor de TNF e IL-6 além de aumentar a taxa de translocação bacteriana (32). ZHENGWU et al. (18) e FARBER et al. (33) estudaram a liberação de IL-1 e IL-6 no endotélio intestinal pós isquemia/reperfusão num modelo murino. A permeabilidade intestinal altera-se sensivelmente e a sua alteração é tanto mais acentuada quanto maior for o período de isquemia. WELCH et al. (34) observaram que a incidência de doenças concomitantes, incluindo a disfunção múltipla de órgãos, são muito mais freqüentes em pacientes que apresentam sinais de isquemia intestinal.

A reperfusão dos pulmões e coração também apresenta um aspecto delicado. Estudos sobre as conseqüências da reperfusão no miocárdio introduziu o conceito de lesão por reperfusão. Isto ocorre porque, se de um lado a reperfusão restabelece a oxigenação do miocárdio, causa também uma reação inflamatória local intensa que provoca dano do miocárdio. O ponto de partida para o estabelecimento do dano é o endotélio microvascular.

A cascata das citocinas que media esta reação inicia-se com a ativação do complemento e liberação de TNF, degranulação de mastócitos e liberação de histamina, que induzem a expressão de proteínas da família das selectinas, que atraem leucócitos. A partir daí, leucócitos migram para a região. Para que leucócitos infiltrados afetem os cardiomiócitos, é necessária a expressão da ICAM1 em sua membrana, fazendo com que haja contato entre as células (13,35-39). Isto acontece sob o estímulo das interleucinas, principalmente a IL-6, IL-8, TNF e IL-1. Os leucócitos assim aderidos causam o "burst" oxidativo que, além de destruir os miócitos cardíacos, também destroem a matriz celular e estruturas de outras células (Figura 1). A disfunção em si aparece em cerca de 2.5 minutos após a geração desta "explosão" de radicais superóxido.

Sugere-se a remoção dos leucócitos durante a CEC, na tentativa de reduzir o número destas células ativadas. Isto poderia diminuir a reação inflamatória pós-operatória. De fato, um estudo sobre isto provou que pacientes tinham melhor oxigenação após remoção dos leucócitos, mas não demonstrou nenhum outro ponto positivo e o quadro clínico como um todo permaneceu inalterado ${ }^{(4)}$.

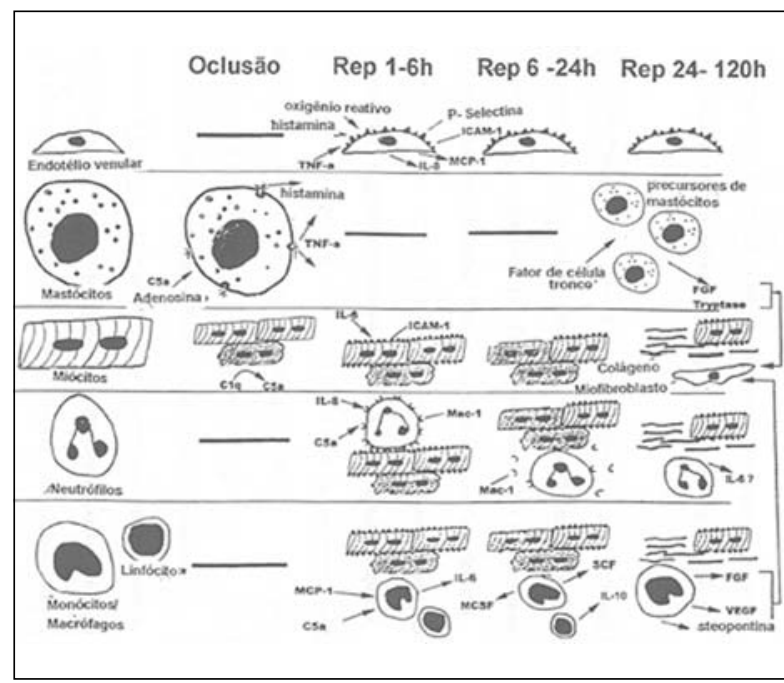

Fig 1- Esquema simplificado dos mecanismos de adesão celular mediado por interleucinas (modificado a partir de Frangogiannis et al. ${ }^{15}$ ).

Um grande número de dados experimentais incluiria também os seguintes danos causados pela reperfusão na microcirculação do miocárdio: capacidade reduzida de vasodilatação endotélio-dependente (disfunção endotelial) e permeabilidade microvascular aumentada $(36,40,41)$.

Os danos à microcirculação do miocárdio têm como citocinas mediadoras principais o TNF e a IL1. O mecanismo proposto para explicar sua ação começa com a geração de espécies reativas do oxigênio (ROS, do inglês: reactive oxygen species), um evento considerado clássico na inflamação. Estas espécies são liberadas por leucócitos ativados (por citocinas) e, por sua vez, aumentam a expressão dessas mesmas citocinas e fatores de adesão celular (selectina -P, ICAM-1).

A síndrome do stress respiratório do adulto é um outro evento relacionado à reação inflamatória sistêmica. A IL-8 não é específica da cirurgia cardíaca mas às situações de isquemia e reperfusão ou de dano associado à anoxia/hiperoxia. Estudos experimentais demonstraram que danos por reperfusão em pulmões poderiam ser evitados com o uso de um anticorpo monoclonal da IL-8. Supõese que a produção dessa interleucina intraalveolarmente seja feita pelos macrófagos. A interação entre os neutrófilos e endotélio alveolar ocorre quando as células do endotélio e os neutrófilos estão ligadas através das moléculas de adesão que se expressam sob a influência das interleucinas. Há, então, liberação de radicais livres de oxigênio e proteases que danificam a microvasculatura pulmonar. O endotélio pulmonar ativado também se torna protrombótico. Tem-se procurado encontrar uma relação entre os níveis de interleucina 1 e TNF encontrados em lavagens broncoalveolares e o grau 
Moura H V, Pomerantzeff P M A, Gomes W J - Síndrome da resposta inflamatória sistêmica na circulação extracorpórea: papel das interleucinas. Rev Bras Cir Cardiovasc 2001; 16(4): 376-87.

da síndrome do stress respiratório. Até o momento, não há resultados conclusivos. A IL-8 não foi medida em estudos desta natureza, provavelmente porque suas concentrações não sejam mensuráveis no momento da coleta da lavagem $(7,11)$.

O cérebro também pode ser afetado pela isquemia durante a CEC. A interleucina mais ativa a nível do sistema nervoso central é a IL-8. Um estudo in vitro ${ }^{(42)}$ delineou a seqüência dos eventos que levam ao dano neuronal por isquemia/reperfusão. Uma vez mais, as células endoteliais estão envolvidas nos primeiros passos do processo. Sob a ação da endotelina1, um vasoconstritor, estas células iniciam a produção de IL-8 que atrai as células polimorfonucleares responsáveis pelo ataque aos neurônios.

No fígado, os efeitos da isquemia/reperfusão iniciam-se com a ativação das células de Kupfer, que então liberam mediadores pró-inflamatórios, tais como o TNF. Estes mediadores estimulam a liberação das citocinas quimiotáticas (ou quimiocinas): IL-1, IL-6, IL-8 e de moléculas de adesão vascular, que mediam a adesão entre as células endoteliais e os neutrófilos atraídos para a região pelas citocinas e outros fatores. A reperfusão aumenta a expressão do TNF e da proteína inflamatória MIP-2 dos macrófagos. O quadro do dano hepático por isquemia/reperfusão segue os mesmos passos do dano no miocárdio. Um sinal identificável desta reação está no edema do fígado, que pode ocorrer durante o processo.

Poderá ainda ocorrer disfunção múltipla de órgãos em detrimento à isquemia, reperfusão com liberação de complemento e anafilotoxinas, produzidas com o uso da CEC.

\section{Imunomodulação Perioperatória das Citocinas}

Agentes anestésicos. Já se tem conhecimento amplo da resposta das citocinas durante procedimentos cirúrgicos em geral pelo fato de que a anestesia causa uma depressão generalizada do sistema imune. Mas é nas cirurgias cardíacas que suas concentrações têm uma variação estatisticamente significativa. Mesmo quando não se pode identificar alguma concentração mensurável de certas citocinas pró-inflamatórias (por exemplo, IL-1 e IL-8) no plasma durante cirurgia cardíaca, lisatos de leucócitos acusaram sua presença depois da CEC (15). Esta resposta sempre foi atribuída ao stress operatório, mas LAHAT et al. (31), no começo dos anos 90 , foram os primeiros a observar uma elevação significativa do TNF imediatamente após a indução de uma dose alta de fentanil, embora não tenham feito nenhuma observação sobre a pertinência do fato. Igualmente, 10 minutos após a anestesia, observa-se um aumento significativo da concentração da IL-8, mesmo antes que a entubação e a cirurgia comecem. Um pequeno aumento de concentração das citocinas inflamatórias pós indução anestésica não está associado a efeitos adversos.
É importante notar que, em uma cirurgia cardíaca, esta reação é seguida de uma resposta anti-inflamatória com liberação de IL-10 seguida de liberação de IL-1ra e de receptores solúveis do TNF.

O conceito de modulação perioperatória das citocinas pelos agentes anestésicos é exemplificado pela IL-6. Ela é liberada sempre em resposta a uma cirurgia de porte e está associada a morbidade pósoperatória. CROZIER et al. (43) mostraram que anestesia de propofol- alfentanil retarda e diminui a magnitude da resposta da IL-6, em comparação ao isoflurano durante grandes cirurgias abdominais. Eles sugerem que este efeito se deve ao fato de que o alfentanil age sobre os receptores opióides levando a uma redução da adenosina monofosfato cíclica intracelular (cAMP), que é um mensageiro secundário importante para a indução da IL-6.

Estudos in vitro mostraram que a produção de TNF ocorre com propofol, tiopental e ketamina. IL1 foi mais induzida com propofol e a IL-6 com ketamina. Um estudo clínico mais recente, entretanto, mostrou que a ketamina, numa única dose, seria um bom supressor da IL-6 A IL-4 foi elevada após administração de tiopental, ketamina e propofol (19), e também protamina ${ }^{(9)}$. Um resumo dos estudos sobre 0 assunto está na Tabela 1 .

\section{Técnicas da CEC}

Embora tendo muitas vantagens na teoria, os circuitos revestidos de heparina não mostraram grandes vantagens durante cirurgias cardíacas. Especificamente, com relação ao nível das citocinas e do complemento, alguns estudos não mostraram nenhuma evidência significativa de mudança dele (9) com o uso de circuitos heparinisados ou não. Alguns investigadores observaram níveis plasmáticos menores de citocinas em pacientes submetidos a CEC com um circuito revestido de heparina (44). Ainda assim, nenhuma mudança positiva no quadro clínico destes pacientes foi observada.

O bombeamento do sangue por vórtice centrífugo tem mostrado uma diminuição na ativação do complemento e dos neutrófilos assim como da hemólise. Entretanto, não causou diminuição estatisticamente significante das citocinas em pacientes pediátricos que tiveram este tipo de bomba na CEC.

O uso de oxigenadores de membrana na redução de complicações inflamatórias também é controvertido. Alguns estudos mencionam diminuição da ativação do complemento enquanto outros não observam nenhuma mudança importante neste aspecto.

Também há controvérsia se a hipotermia e a normotermia durante a CEC têm alguma influência no quadro clínico. A hipotermia parece reduzir marcadores da ativação do complemento segundo alguns estudos e MENASCHÉ et al. $(20,45)$ demonstraram que a hipotermia reduziu marcadores de 
Moura H V, Pomerantzeff P M A, Gomes W J - Síndrome da resposta inflamatória sistêmica na circulação extracorpórea: papel das interleucinas. Rev Bras Cir Cardiovasc 2001; 16(4): 376-87.

Tabela 1

\begin{tabular}{|c|c|c|c|}
\hline \multicolumn{4}{|c|}{ Efeito de diferentes anestésicos na produção das citocinas } \\
\hline Estudo & Cirurgia & Droga/Técnica & Efeito \\
\hline $\begin{array}{l}\text { Brix-Christensen } \\
\text { et al. }\end{array}$ & CABG com CEC & $\begin{array}{l}\text { epidural e anestésico } \\
\text { inalável } X \text { alta dose } \\
\text { de fentanil }\end{array}$ & $\begin{array}{l}\text { IL-6, IL-8 e IL-10 tiveram } \\
\text { respostas similares com as } \\
\text { duas técnicas }\end{array}$ \\
\hline Taylor et al. & Pélvica & $\begin{array}{l}3 \text { ou } 15 \mathrm{mg} / \mathrm{kg} \text { de fentanil } \\
\text { suplementando halotano }\end{array}$ & $\begin{array}{l}\text { diferença na concentração } \\
\text { de IL-6 NS* }\end{array}$ \\
\hline Crozier et al. & Histerectomia & $\begin{array}{l}\text { alfentanil e propofol X } \\
\text { fentanil, metohexitone e } \\
\text { isofluorano }\end{array}$ & $\begin{array}{l}\text { maior liberação de IL- } 6 \text { em } \\
\text { menos tempo no segundo grupo }\end{array}$ \\
\hline Norman e Fink & $\begin{array}{l}\text { Substituição da } \\
\text { aorta abdominal }\end{array}$ & $\begin{array}{l}\text { geral c/ morfina no pós-op. X } \\
\text { regional com } \\
\text { bupivacaína/geral e morfina } \\
\text { epidural no pós-op. }\end{array}$ & $\begin{array}{l}\text { diferença dos níveis de IL-1, } \\
\text { IL-6 e TNF NS entre grupos }\end{array}$ \\
\hline Moore et al. & Histerectomia & geral X geral/extradural & $\begin{array}{l}\text { diferença na concentração } \\
\text { de IL-6 NS** }\end{array}$ \\
\hline \multicolumn{4}{|l|}{ Roytblat et al. } \\
\hline Hiesmayr et al. & $\begin{array}{l}\text { Cardiotorácica } \\
\text { com CEC X sem CEC }\end{array}$ & $\begin{array}{l}\text { geral } \\
\text { altos no grupo que teve CEC }\end{array}$ & níveis de IL-6 e IL-10 mais \\
\hline Jameson et al. & Histerectomia & etomidato $\mathrm{X}$ tiopental & $\begin{array}{l}\text { nível de IL- } 6 \text { mais alto com } \\
\text { etomidato }\end{array}$ \\
\hline
\end{tabular}

${ }^{*} \mathrm{NS}=$ Não significante

inflamação como o TNF e a IL-6, assim como reduziu a ativação de neutrófilos. Outros estudos, todavia, não encontraram nenhuma associação entre normotermia e hipotermia para estes marcadores, sugerindo que deve haver um limite para a ação da temperatura isoladamente na ativação das citocinas.

Terapias em Potencial para Prevenção e Tratamento das Complicações Relacionadas à Reação Inflamatória Sistêmica

Atualmente, não existem terapias usadas em larga escala para prevenir ou tratar disfunção de órgão resultante da inflamação sistêmica. O que parece mais lógico é tentar intervenções a nível dos mediadores da inflamação. Neste sentido, houve tentativas de emprego dos receptores antagonistas de certas interleucinas sem muito sucesso. Ou a terapia não surtiu efeitos mensuráveis, ou ela demonstrou ser mais arriscada que benéfica, como no caso do uso do receptor antagonista da IL-1, com a qual se observou uma mortalidade maior que a normal.

Aceita-se o fato de que a endotoxemia é prevalente em cirurgias cardíacas. Desta maneira, as terapias que visam diminuir seus efeitos são uma opção em estudo. Estas estratégias pressupõem que a incidência de endotoxemia durante cirurgias cardíacas é causada pela translocação de LPS através das paredes do intestino isquêmico. As possibilidades incluem a descontaminação digestiva seletiva e a otimização do volume intravascular.
Drogas, como a pentoxifilina (46), indometacina, ácido aminocapróico e aprotinina também têm sido usadas com o intuito de reduzir a resposta inflamatória causada pela CEC, conseqüentemente, buscando diminuir as interleucinas circulantes já citadas.

Uso de esteróides. O uso de esteróides tem ganhado popularidade na prevenção da elevação das citocinas pró-inflamatórias durante cirurgias cardíacas. Num estudo de JANSEN et al. (21), onde se administrou dexametasona aos pacientes (na indução da anestesia), preveniu-se o aumento do TNF, assim como reduziu-se a hipertermia e a hipotensão pós-operatórias. Observou-se uma tendência à melhora do quadro clínico dos pacientes. Outros detectaram uma elevação minimizada da IL-6, IL-1 e IL-8 (31) sem, entretanto, mostrar alguma vantagem na evolução do quadro clínico do paciente.

Resultados positivos mais evidentes clinicamente têm sido obtidos com metilprednisolona. A função pulmonar parece beneficiar-se do uso pré-operatório deste esteróide $(4,31,32,47,48)$.

FEY et al. (49) observam que havia uma diminuição da depressão miocárdica em função da isquemia, onde essas drogas agem estabilizando a membrana dos lisossomos com conseqüente diminuição da autólise celular e havendo aumento de células miocárdicas visíveis.

JANSEN et al. (21) demonstram que o uso da dexametasona profilaticamente em operação de 
Moura H V, Pomerantzeff P M A, Gomes W J - Síndrome da resposta inflamatória sistêmica na circulação extracorpórea: papel das interleucinas. Rev Bras Cir Cardiovasc 2001; 16(4): 376-87.

revascularização do miocárdio com CEC reduz a liberação de TNF-a, do leucotrieno b4 e também do fator ativador de plasminogênio. ENGELMAN et al. (47) relataram que a utilização profilática de metilprednisolona e dexametasona em pacientes submetidos à operação cardíaca com CEC, pode produzir uma diminuição acentuada dos níveis de complemento e também de citocinas, com ausência de reações adversas devido ao uso de esteróides.

TABARDEL et al. (50) demonstraram que a administração profilática de corticóide diminui a liberação de interleucinas pró-inflamatórias e também aumenta a liberação de IL-10 que é uma interleucina anti-inflamatória.

Em seu estudo, BRASIL et al. (46) concluíram que a metilprednisolona inibe significativamente a liberação de citocinas pró-inflamatórias, principalmente o TNF, e os efeitos sistêmicos pós CEC foram alterados. Resultados positivos com metilprednisolona foram encontrados em outros estudos, onde a função pulmonar parece beneficiar-se do uso préoperatório deste esteróide $(4,31,32,47,48)$.

Podemos concluir que o uso dos esteróides diminuei os efeitos da resposta inflamatória encontrada no pós-operatório com CEC. Não foi encontrado, ao longo das referências citadas, a determinação definitiva das doses que devem ser utilizadas para os esteróides citados; acredita-se que algum estudo possa estar sendo conduzido com este objetivo.

\section{CONCLUSÃO}

Com a leitura destes trabalhos, fica evidente o aumento das interleucinas circulantes nas cirurgias cardíacas realizadas com o uso da circulação extracorpórea (CEC), como resultado do contato do sangue com a superfície não endotelial, levando a uma cascata de reações inflamatórias com conseqüências trans-cirúrgicas e pós-cirúrgicas.

Estas reações sempre acontecem, em menor ou maior grau, são mais evidentes e deletérias em idosos e neonatos, podendo manifestar-se uma febre ou até mesmo levar à morte o paciente.

As interleucinas ditas pró- inflamatórias, IL-1, IL-6, IL-8, são consideradas as 'vilãs' desse processo, nele participando de forma direta; as condições por elas causadas são tanto mais complicadas quanto maior for o nível dessas citocinas no organismo, fato que poderá ser diminuído com o uso de drogas, como já demonstrado em trabalhos e com medidas profiláticas já referidas. 0 aumento natural da IL-10, não- inflamatória, que age como antagonista das outras, já citadas, diminui as várias facetas da SIRS e seus danos.

RBCCV 44205-564

Moura H V, Pomerantzeff P M A, Gomes W J - Systemic inflammatory response syndrome: role of the interleukins. Rev Bras Cir Cardiovasc 2001; 16(4): 376-87.

ABSTRACT: The systemic inflammatory response syndrome (SIRS) occurs in the cardiac surgery more often with the use of CPB. It is due to the contact of the blood with non-endothelial surfaces and, later to reperfusion. This contact leads to the release of substances such as anaphylotoxins, which are activated by the complement factor, and stimulate the production of pro-inflammatory cytokines (IL1, IL-6, TNF-a). Cytokines are responsible for induction of fever, neutrophilia with increase of adhesiveness between neutrophils and mocytes and, they act synergetically stimulating the production of other interleukins (IL-6, IL-8). It is observed that intestinal ischemia secondary to CPB, causes the production of IL-6, TNF-a, IL-1 in the intestinal epithelium and, reperfusion post CPB induces the expression of IL-1 and TNF-a in the vascular endothelium that can generate a dysfunction able to injure cardiomyocytes. Following the post-CPB cascade of reactions, the adult respiratory distress syndrome is mediated by IL-8, found the at alveolar level. The brain and the liver can also be affected by ischemia/reperfusion phenomena and, once more, it is shown that interleukins (IL-8 and IL-13, respectively) have an important role in the course of SIRS- related events. CPB and anesthesia techniques can influence interleukin activity. There is not a consensus regarding preventive or treatment therapies for complications from SIRS. Possible choices are: intervention at the level of inflammation mediators, digestive decontamination procedures, use of drugs such as indometacin or steroids. The regulation of the inflammatory process, generally depends on the balance between anti- and pro- inflammatory cytokines. These later are detectable in normal individuals whereas the others only manifest locally or are almost inexistent. We conclude that the increase of the concentration of pro-inflammatory interleukins in the organism is expected during important surgical intervention. Such an increase will bring complications if it is exacerbated and, the organism is debilitated.

DESCRIPTORS: Extracorporeal circualtion, adverse effects. Interleukins. Cardiac surgical procedures, adverse effects. 
Moura H V, Pomerantzeff P M A, Gomes W J - Síndrome da resposta inflamatória sistêmica na circulação extracorpórea: papel das interleucinas. Rev Bras Cir Cardiovasc 2001; 16(4): 376-87.

AGRADECIMENTOS: A Deus, pois sem Ele nada somos. Aos Profs. Drs. Pablo Maria A Pomerantzeff e Walter José Gomes. Aos perfusionistas Antônio Gilberto Martins, Benedito dos Santos, Adriana Noronha e Rosimeire Castilhioni Branco, e à amiga e Profa. de Inglês Bartira Mendes-Soares.

\section{REFERÊNCIAS BIBLIOGRÁFICAS}

1 Stammers A H - Historical aspects of cardiopulmonary bypass: from antiquity to acceptance. $J$ Cardiothorac Vasc Anesth 1997; 11: 266-74.

2 Souza M H L \& Elias D O - Circulação extracopórea: histórico e desenvolvimento. In: Fundamentos da circulação extracorpórea. Rio de Janeiro, Centro Editorial Alfa Rio, 1995: V. 1: 1-27.

3 Muckart D J \& Bhagwanjee S - American College of Chest Physicians/Society of Critical Care Medicine Consensus Conferenec definitions of the systemic inflammatory response syndrome and allied disorders in relation to critically injured patients. Crit Care Med 1997; 25: 1789-95.

4 Bennett-Guerrero E - Systemic inflammation. In: Cardiac Anesthesia. Kaplan J A, ed. Philadelphia: W. B. Saunders, 1999: 297-318.

5 Souza M H L \& Elias D O - Resposta inflamatória sistêmica à circulação extracorpórea. Perfusion on line www.perfline.com, 1999.

6 Silva S L - Síndrome de resposta inflamatória sistêmica pós circulação extracorpórea. In: Temas atuais em circulação extracorpórea. Teixeira Filho G F et al., eds. Sociedade Brasileira de Circulação Extracorpórea, 1997: 90-7.

7 Hairston $\mathrm{P}$, Manos J P, Graber C D, Lee WH Jr. Depression of immunologic surveillance by-pumpoxygenation perfusion. J Surg Res 1969; 9: 587-93.

8 Parker D J, Cantrell G, Stroud R, Karp R B, Digerness $S$ B - Changes in serum complement and immunoglobulins following cardiopulmonary bypass. Surgery 1972; 71: 824-7.

9 Steinberg J B, Kapelanski D P, Olson J D, Weiler J M - Cytokine and complement levels in patients undergoing cardiopulmonary bypass. J Thorac Cardiovasc Surg 1993; 106: 1008-16.

10 Chenoweth D E; Cooper S W; Hugli T E; Stewart R W; Blackstone E H; Kirklin J W - Complement activation during cardiopulmonary bypass: evidence for generation of $\mathrm{C} 3 \mathrm{a}$ and $\mathrm{C} 5 \mathrm{a}$ anaphylatoxins. $\mathrm{N}$ Engl J Med 1981; 304: 497-503.

11 Butler J; Rocker G M, Westaby S - Inflammatory response to cardiopulmonary bypass. Ann Thorac Surg 1993; 55: 552-9.
12 Mayers I \& Johnson D - The nonspecific inflammatory response to injury. Can J Anaesth 1998; 45: 871-9.

13 Frangogiannis N G, Youker K A; Rossen R D et al. Cytokines and the microcirculation in ischemia and reperfusion. J Mol Cell Cardiol 1998; 30:2567-76.

14 Yoshidome $\mathrm{H}$, Kato A, Miyazaki M, Edwards M J, Lentsch A B - IL-13 activates STAT6 and inhibits liver injury induced by ischemia/reperfusion. Am J Pathol 1999; 155:1059-64.

15 Cal RGR - Trauma celular em CEC e suas conseqüências. In: Andrade JCS ed., São Paulo, 1988; Circulação extracorpórea. São Paulo: Biotecno, sd.

16 Knudsen F \& Andersen LW - Immunological aspects of cardiopulmonary bypass. J Cardiothorac Anesth 1990; 4: $245-58$.

17 Ferroni P, Speziale G, Ruvolo G et al. - Platelet activation and cytokine production during hypothermic cardiopulmonary bypass: a possible correlation? Thromb Haemost 1998; 80: 58-64.

18 Sun Z, Wang X, Lasson $\mathrm{H}$ et al. - Roles of plateletactivator factor, interleukin-1 beta and interleukin- 6 in intestinal barrier dysfunction induced by mesenteric arterial ischemia and reperfusion. J Surg Res 1999; 87: $90-100$.

19 Haeffner-Cavaillon N, Roussellier N, Ponzio O - Induction of Interleukin-1 production in patients undergoing cardiopulmonary bypass. J Thorac Cardiovasc Surg 1989, 98: 1100-6.

20 Menasché $\mathrm{P}$, Haydar S, Peynet $\mathrm{J}$ et al. - A potential mechanism of vasodilation after warm heart surgery. J Thorac Cardiovasc Surg, 1994; 107: 293-9.

21 Jansen $\mathrm{N}$ J, van Oeveeren $\mathrm{W}, \mathrm{Gu} \mathrm{Y} J$, van Vliet $\mathrm{M} \mathrm{H}$, Eijsman L, Wildevuur C R - Endotoxin release and tumor necrosis factor formation during cardio-pulmonary bypass. Ann Thorac Surg 1992; 54: 744-8.

22 Roth-Isigkeit A, Borstel TV, Seyfarth M, Schmucker P Perioperative serum levels of tumor-necrosis-factor alpha (TNF-alpha), IL-1 beta, IL-6, IL-10 and soluble IL-2 receptor in patients undergoing cardiac surgery with cardiopulmonary bypass without and with correction for haemodilution. Clin Exp Immunol 1999; 118:242-6.

23 Beutler B \& Cerami A - Tumor necrosis, cachexia, shock, and inflammation: a common mediator. Ann Ver Biochem 1988; 57: 505-18.

24 Casey LC - Role of the cytokines in the pathogenesis of cardiopulmonary-induced multisystem organ failure. Ann Thorac Surg 1993; 56: (5 Suppl) S92-6.

25 Cain B S, Meldrum D R, Dinarello C A et al. - Tumor necrosis factor-alpha and interleukin-1 beta synergistically depress human myocardial function. Crit Care Med 1999; 27:1309-18. 
Moura H V, Pomerantzeff P M A, Gomes W J - Síndrome da resposta inflamatória sistêmica na circulação extracorpórea: papel das interleucinas. Rev Bras Cir Cardiovasc 2001; 16(4): 376-87.

Hauser G J, Bem-Ari J, Colvin MP et al. - Interleukin6 levels in serum and lung lavage fluid of children undergoing open heart surgery correlate with postoperative morbidity. Intensive Care Med 1998; 24: $481-6$.

Cremer J, Martin M, Redl H et al.- Systemic inflammatory response syndrome after cardiac operations. Ann Thorac Surg 1996; 61: 1714-20.

Frering B, Philip I, Dehoux M, Rolland C, Langlois JM, Desmonts J M - Circulating cytokines in patients undergoing normothermic cardiopulmonary bypass. $J$ Thorac Cardiovasc Surg 1994; 108: 636-41.

Campos A H \& Santos B F C- O papel das citocinas na disfunção ventricular avançada. Rev Soc Cardiol Estado de São Paulo 1998; 8: 400-5.

30 Hill G E, Pohorecki R, Whitten C W - Plasma lipid concentrations correlate inversely with CPB-induced interleukin-6 release. Can J Anaesth 1998; 45:50914.

McBride W T, Armstrong M A, McBride S J Immunomodulation: an important concept in modern anaesthesia. Anaesthesia 1996; 51: 465-73.

Khew-Goodall Y, Wadham C, Stein B N, Gamble J R, Vadas M A - Stat6 activation is essential for interleukin-4 induction of $\mathrm{P}$-selectine transcription in human umbilical vein endothelial cells. Artherioscler Thromb Vasc Biol 1999; 19:1421-9.

33 Farber A, Connors J P, Friedlander R M, Wagner R J, Powell R J, Cronenwett $\mathrm{J} L$ - A specific inhibitor of apoptosis decreases tissue injury after intestinal ischemia-reperfusion in mice. J Vasc Surg 1999; 30: 752-60.

Welch M, Baguneid M S, McMahon R F et al. - Histological study of colonic ischemia after aortic surgery. $\mathrm{Br} \mathrm{J}$ Surg 1998; 85:1095-8.

Grotz M; Regel G, Schratt H E, Seekamp A, Pape H C, Tscherne $\mathrm{H}$ - Intestinal cytokine liberation after intestinal ischemia in the rat: studies in the Ussing chamber system. Z Gastroenterol 1996; 34:783-90.

Barros L F M, Petrini C A, Chagas A C - Isquemia e reperfusão: a acumulação de leucócitos no miocárdio. Instituto do Coração, Comunicação Interna.

37 Simms MG \& Walley KR- Activated macrophages decrease rat cardiac myocyte contractility: importance of ICAM-1-dependent adhesion. Am J Physiol 1999; 277(1 Pt 2):H253-60.

38 Seekamp A, Jochum M, Ziegler M, van Griensven M, Martin M, Regel G - Cytokines and adhesion molecules in elective and accidental trauma-related ischemia/reperfusion. J Trauma 1998; 44: 874-82.
39 Frangogiannis N G, Lindsey M L, Michael L H et al. Resident cardiac mast cells degranulate and release performed TNF-alpha, initiating the cytokine cascade in experimental canine myocardial ischemia/ reperfusion. Circulation 1998; 98: 699-710.

40 Cameron D - Initiation of white cell activation during cardiopulmonary bypass: cytokines and receptors. $J$ Cardiovasc Pharmacol 1996; 27(Suppl 1):S1-5.

41 Hansen P R - Inflammatory alterations in the myocardial microcirculation. J Mol Cell Cardiol 1998; 30: 25559.

42 Hofman FM, Chen P, Jeyaseelan R, Incardona F; Fisher $M$, Zidovetzki R - Endothelin-1 induces productionof the neutrophil chemotactic factor interleukin- 8 by human brain-derived endothelial cells. Blood 1998; 92: 3064-72.

43 Crozier T A, Müller J E, Quittkat D et al. - Effect of anaesthesia on th cytokine responses to abdominal surgery. Br J Anaesth 1994; 72: 280-5.

44 Wan S, Leclerc J L Antoine M, Desmet J M, Yim A P, Vincent $J \mathrm{~L}$ - Heparin-coated circuits reduce miocardial injury in heart or heart-lung transplantation: a prospective, randomized study. Ann Thorac Surg $1999 ; 68: 1230-5$

45 Menasché $P$, Peynet $J$, Larivière $J$ et al. - Does normothermia during cardiopulmonary bypass increase neutrophil-endothelium interactions? Circulation 1994, 90 (5 Pt 2): II275.

46 Brasil L A, Gomes W J, Salomão R, Fonseca J H P, Branco J N R, Buffolo E - Uso de corticóide como inibidor de resposta inflamatória induzida pela circulação extracorpórea. Rev Bras Cir Cardiovasc 1999, 14: $254-68$.

47 Engelman R M, Rousou J A, Flack J E $3^{\text {rd }}$, Deaton D W, Kalfin R, Das D K- Influence of steroids on complement and cytokine generation after cardiopulmonary bypass. Ann Thorac Surg 1995; 60: 801-4.

48 Lodge A J, Chai P J, Daggett C W, Ungerleider R M, Jaggers $J$ - Methylprednisolone reduces the inflammatory response to cardiopulmonary bypass in neonatal piglets: timing of dose is important. $J$ Thorac Cardiovasc Surg 1999; 11: 515-22.

49 Fey K, Follette D, Livesay J et al. - Effects of membrane stabilization on the safety of hypothermic arrest after aortic cross-clamping. Circulation 1977; 56 (3 Suppl II): 143-7.

50 Tabardel Y, Duchateau J, Schmartz D et al. Corticosteroids increase blood interleukin-10 levels during cardiopulmonary bypass in men. Surgery 1996; 119: $76-80$. 